GRASAS Y ACEITES 70 (2)

April-June 2019, e303

ISSN-L: 0017-3495

https://doi.org/10.3989/gya.0698181

\title{
Effect of heating on the oxidative stability of corn oil and soybean oil
}

\author{
R. Saeed ${ }^{\mathrm{a}}$ and S. Naz ${ }^{\mathrm{a}, \bigotimes}$ \\ ${ }^{a}$ Department of Chemistry, University of Karachi, Karachi 75270, Pakistan \\ ${ }^{\square}$ Corresponding author: sam_cancer90@yahoo.com
}

Submitted: 24 June 2018; Accepted: 15 October 2018; Published online: 14 February 2019

SUMMARY: The effects of conventional and microwave heating on the oxidative properties of corn and soybean oil were evaluated. The results showed that acid value, peroxide value, oxidative indices, total oxidation value, and $p$-anisidine values changed significantly with the rise in temperature $(\mathrm{p}<0.05)$. The peroxide and $p$-anisidine values for corn oil (PV: $50.670 \mathrm{meqO}_{2} / \mathrm{kg}, p$-AV: 8.248 ) were greater than soybean oil (PV: 41.694 $\mathrm{meqO}_{2} / \mathrm{kg}, p$-AV: 7.566 ) for conventional heating. The peroxide and $p$-anisidine values for soybean oil (PV: 6.545 $\mathrm{meqO}_{2} / \mathrm{kg}, p$-AV: 76.539 ) were greater compared to corn oil ( $\mathrm{PV}: 5.074 \mathrm{meqO}_{2} / \mathrm{kg}, p$-AV: 65.360 ) for microwave heating. The results concluded that microwave heating had a greater impact on the chemical degradation of the fatty acids of the oil. The FT-IR spectra showed peak changes at $3743 \mathrm{~cm}^{-1}$ and $1739 \mathrm{~cm}^{-1}$ and confirmed the rancidity of the oils from microwave heating due to the formation of secondary oxidation products. It was concluded that corn oil showed more oxidative changes compared to soybean oil.

KEYWORDS: Corn and soybean oil; FT-IR analysis; Heating; Oxidative stability

RESUMEN: Efecto del calentamiento sobre la estabilidad oxidativa de aceites de maíz y de soja. Se ha evaluado el impacto del calentamiento convencional y por microondas en las propiedades oxidativas de aceites de maíz y de soja. Los resultados mostraron que con el aumento de la temperatura, el índice de acidez, de peróxido, los índices de oxidación, el valor de oxidación total y los valores de $p$-anisidina cambiaron significativamente ( $\mathrm{p}<0.05$ ). Los valores de peróxido y $p$-anisidina del aceite de maíz (PV: 50,670 meq $\mathrm{O}_{2} / \mathrm{kg}, \rho-\mathrm{AV}: 8.248$ ) fueron mayores que para el aceite de soja (PV: 41,694 meqO $/ 2 \mathrm{~kg}, p$-AV: 7.566) para el calentamiento convencional. Los valores de peróxido y $p$-anisidina del aceite de soja ( $\mathrm{PV}: 6,545 \mathrm{meqO}_{2} / \mathrm{kg}, p$-AV: 76,539) fueron mayores en comparación con el aceite de maíz (PV: 5,074 meqO $2 / \mathrm{kg}, p$-AV: 65,360) para el calentamiento por microondas. Los resultados concluyeron que el calentamiento por microondas tuvo más impacto en la degradación química de los ácidos grasos de los aceites. Los espectros FT-IR mostraron cambios máximos a $3743 \mathrm{~cm}^{-1}$ y $1739 \mathrm{~cm}^{-1}$, también confirmaron la rancidez de los aceites en el calentamiento por microondas mediante la formación de productos de oxidación secundarios. Se concluyó que el aceite de maíz mostraba más cambios oxidativos en comparación con el aceite de soja.

PALABRAS CLAVE: Aceites de maíz y de soja; Análisis FT-IR; Calentamiento; Estabilidad oxidativa

ORCID ID: Naz S https://orcid.org/0000-0002-6729-9532, Saeed R https://orcid.org/0000-0001-5302-2017

Citation/Cómo citar este artículo: Naz S, Saeed R. 2019. Effect of heating on the oxidative stability of corn oil and soybean oil. Grasas Aceites $\mathbf{7 0}$ (2), e303. https://doi.org/10.3989/gya.0698181

Copyright: (C2019 CSIC. This is an open-access article distributed under the terms of the Creative Commons Attribution 4.0 International (CC BY 4.0) License. 


\section{INTRODUCTION}

The oxidative stability of oil is affected not only by a change in temperature, but also by using different heating techniques and extraction processes (Bakhshabadi et al., 2018 and 2017, Taghvaei et al., 2015). Prolonged heating and frying processes are generally used for the preparation of food at home and in industries. Vegetable oils consist of molecules of esters derived from glycerol and have a different degree of unsaturation. Fatty acids like linoleic and linolenic acid and the fat-soluble vitamins (A, D, E, and $\mathrm{K}$ ) of vegetable oils are the source of energy to the human body for better growth and healthy organs (El-Hadad et al., 2010; Aluyor and Ori-Jesu, 2008).

The heating of oils at high temperature produces changes in the fatty acid constituents of triglyceride molecules, which alters the physicochemical properties of vegetable oil due to the change in chain length, unsaturation degree and position of unsaturation (Fasina et al., 2006).

The rapid cooking and and heating of food using a microwave is a common practice at the domestic level due to the ease of its usage and time-saving method (Caponio et al., 2003). Microwave transmits heat that has high penetration power which reduces the time, effort and energy but creats serious health issues (Abbas et al., 2016; Abbas et al., 2017). Microwave irradiation causes changes in the chemical composition of edible oil during the process of hydrolysis, oxidation and polymerization reactions which produce rancid flavors and odor in the edible oil (Zahir et al., 2017; Li et al., 2018). The oxidative stability can be achieved by adding natural antioxidants to edible oil (Rafiee et al., 2012; Taghvaei et al., 2014).

Many studies have been carried out to assess the influence of conventional heating and microwave heating on the oxidative stability of edible oils. Sadoudi et al., 2014 found that thermal heating sunflower oil at $99 \pm 2{ }^{\circ} \mathrm{C}$, causes a high level of deterioration by losing a significant amount of essential fatty acids (linoleic acid) with the development of oxidative rancidity. Adejumo et al., 2015, worked on tiger nut oil extraction using thermal heating at $100{ }^{\circ} \mathrm{C}$ and found that heating decreased the oil yield, acid value, free fatty acid, peroxide value, and density. Several research studies reported the effect of microwave heating on physicochemical properties by measuring primary and secondary oxidation products, conjugated products, and fatty acids (Hussain et al., 2015; Abbas et al., 2016; Saeed et al., 2014; Ghosh et al., 2014; Li et al., 2018; Aydinkaptan and Mazi, 2017). Lukesova et al., 2009 studied the oxidative changes which occurred during the microwave heating of vegetable oils and found rapeseed oil to be the most suitable vegetable oil among soybean oil, corn oil and sunflower oil due to the fewer changes observed in conjugated dienes and peroxide value.
The current study was carried out to monitor the degradation of the triglycerides in corn oil and soybean oil upon conventional heating and microwave heating which reduce the physical and chemical characterizations of edible oils.

\section{MATERIALS AND METHODS}

Potassium iodide (KI) from Merck (Darmstadt, Germany), hydrochloric acid $(\mathrm{HCl})$, sodium thiosulphate $\left(\mathrm{Na}_{2} \mathrm{~S}_{2} \mathrm{O}_{3} \cdot 5 \mathrm{H}_{2} \mathrm{O}\right)$, iso-octane $(2,2,4$-trimethylpentane) from Riedel-de Haë, Sigma-Aldrich (Buchs, Switzerland), ethanol $\left(\mathrm{C}_{2} \mathrm{H}_{5} \mathrm{OH}\right)$, isopropanol $\left(\mathrm{C}_{3} \mathrm{H}_{7} \mathrm{OH}\right)$, trichloromethane $\left(\mathrm{CHCl}_{3}\right)$, para-anisidine reagent $\left(\mathrm{C}_{7} \mathrm{H}_{9} \mathrm{ON}\right)$, glacial acetic acid $\left(\mathrm{CH}_{3} \mathrm{COOH}\right)$, potassium hydroxide $(\mathrm{KOH})$ from BDH (England) were used for analysis. All of the reagents and solvents used were of analytical reagent grade.

One kilogram of corn and soybean seeds was purchased on September 2014 from a local market in Karachi, Pakistan. The oil was extracted from the seeds using an expeller machine (HDC, Model LTP205, China). The extracted oils were filtered and stored in sealed glass bottles in the refrigerator (4 ${ }^{\circ} \mathrm{C}$ ) for further analysis.

\subsection{Thermal treatments}

Samples of corn and soybean oil $(50 \mathrm{~mL})$ were heated in Pyrex glass beakers from $303{ }^{\circ} \mathrm{K}$ to $343{ }^{\circ} \mathrm{K}$ using a hot plate $78 \mathrm{HW}-1$ Jiangsu, China (Mainland). Samples were taken at intervals of $10^{\circ} \mathrm{K}$ in separate glass vials and stored until analysis.

Samples of corn and soybean oil $(50 \mathrm{~mL})$ were taken in Pyrex glass beakers and exposed to microwave irradiation (model DW-112 C, Pakistan) operating at the high power setting $(800 \mathrm{~W}, 2450 \mathrm{MHz})$ for $0.5 \mathrm{~min}, 1 \mathrm{~min}, 2 \mathrm{~min}, 4 \mathrm{~min}, 6 \mathrm{~min}$ and $10 \mathrm{~min}$. Samples were collected at each time interval. The temperature of each oil sample was noted using a thermometer. After each heating treatment, the microwave oven was stopped for 30 minutes to cool before starting the next heating. The collected samples were stored in sealed vials until further analysis. The temperature profiles of the oils after microwave irradiation exposure at different heating times are shown in Figure 1.

\subsection{Density (d) and viscosity (n)}

The density of corn oil and soybean oil samples was measured using a relative density bottle and Sartorius electronic balance (model BL-1505, Germany) with $\pm 0.001 \mathrm{~g}$ uncertainty. The viscosity of corn oil and soybean oil samples was determined using Ostwald viscometer (techniconominal constant $0.05 \mathrm{Cs} / \mathrm{c}$, ASTMAD 445 England). The oil sample was poured into a viscometer and the time 


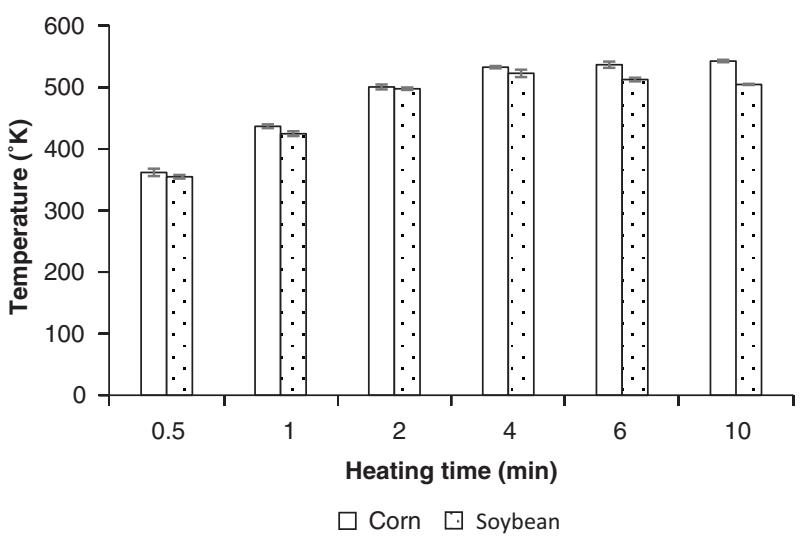

FIGURE 1. Impact of microwave heating time on the temperature of corn and soybean oil. Data are presented as the mean of triplicates with error bars denoting standard deviation.

of flow between the two marks of oil samples was measured using a stopwatch with a count of at least 0.2s. (Zahir et al., 2017).

\subsection{Oxidative indices}

The acid value of the oil samples $(\mathrm{S})$ was measured by dissolving $4 \mathrm{~g}$ oil in $50 \mathrm{~mL}$ 2-propanol and titrated with a $0.1 \mathrm{~N}$ potassium hydroxide solution using a phenolphthalein indicator. A blank (B) determination was also made with the same procedure without the oil sample. The acid value was determined through the reported approach of AOCS Cd 3-63, 1993:

$\mathrm{AV}=(\mathrm{S}-\mathrm{B}) * \mathrm{~N} * 56.11 / \mathrm{g}_{\mathrm{oil}}$

An accurately weighed $1 \mathrm{~g}$ oil sample (S) was dissolved in $15 \mathrm{~mL}$ of a $1 \mathrm{~N}$ ethanolic potassium hydroxide solution $(\mathrm{KOH})$ and $10 \mathrm{~mL}$ double distilled water were added. Refluxed the contents for 40 minutes to convert the triglyceride molecules to glycerol. After cooling, titration was performed with $0.5 \mathrm{~N}$ hydrochloric acid $(\mathrm{HCl})$ using phenolphthalein indicator. Blank (B) determination was also made using same procedure without oil samples. Saponification values were determined through the equation (AOCS Cd 3-25, 1997):

$$
\mathrm{SV}=(\mathrm{B}-\mathrm{S}) * \mathrm{~N} * 56.11 / \mathrm{g}_{\mathrm{oil}}
$$

A $5 \mathrm{~g}$ oil sample (S) was dissolved in a $30 \mathrm{~mL}$ mixture of acetic acid and chloroform which was further reacted with $0.5 \mathrm{~mL}$ of a saturated potassium iodide (KI) solution. After one minute of shaking, $30 \mathrm{~mL}$ water were added and the contents were shaken vigorously to liberate iodine from the organic to the aqueous layer. Iodine was titrated with a $0.1 \mathrm{~N}$ sodium thiosulphate solution using a starch indicator. A blank (B) determination was made using the same procedure without the oil sample. Peroxide values were calculated using the equation (AOCS, 1997 method Cd 8-53):

$\mathrm{PV}=(\mathrm{S}-\mathrm{B}) * \mathrm{~N} * 1000 / \mathrm{g}_{\mathrm{oil}}$

A $5 \mathrm{~g}$ oil sample was dissolved in $25 \mathrm{~mL}$ isooctane. The absorbance of the solution $\left(\mathrm{A}_{\mathrm{b}}\right)$ at 350 $\mathrm{nm}$ was measured using iso-octane as the reagent blank. $5 \mathrm{~mL}$ of the above solution and $5 \mathrm{~mL}$ of iso-octane were pipetted out in separate test tubes which were further diluted with $1 \mathrm{~mL}$ of $p$-anisidine reagent $(2.5 \% \mathrm{w} / \mathrm{v}$ in acetic acid). The absorbance of the sample solution $\left(\mathrm{A}_{\mathrm{s}}\right)$ against the blank was recorded after 10 minutes at $350 \mathrm{~nm}$ using a UV-VIS spectrophotometer (UV-1800 Shimadzu, Koyoto Japan). The $p$-anisidine values for the oil samples were calculated using the equation (AOCS, 1997 method p2.4):

$p-\mathrm{AV}=25\left(1.2 * \mathrm{~A}_{\mathrm{s}}-\mathrm{A}_{\mathrm{b}}\right) / \mathrm{g}_{\mathrm{oil}}$

\subsection{UV-Spectroscopic indices}

The contents of conjugated dienes (CDs) and conjugated trienes (CTs) in terms of specific extinctions at $232 \mathrm{~nm}$ and $268 \mathrm{~nm}$, respectively were monitored using a UV-VIS spectrophotometer (UV-1800 Shimadzu, Koyoto Japan). The absorbances of the oil samples, properly diluted in iso-octane, were recorded and CDs and CTs values were calculated from the following equations (AOAC, 1997 p2.15):

$$
\begin{aligned}
& \text { CDs }=\left(\mathrm{A}_{\mathrm{s}}-\mathrm{A}_{\mathrm{b}}\right) / \mathrm{b} \mathrm{C} \\
& \mathrm{CTs}=\left(\mathrm{A}_{\mathrm{s}}-\mathrm{A}_{\mathrm{b}}\right) / \mathrm{b} \mathrm{C}
\end{aligned}
$$

Where $A_{s}$ and $A_{b}$ is the absorbance of the sample and blank, respectively, $\mathrm{b}$ is the path length and $\mathrm{C}$ represents the percent concentration of oil sample.

\subsection{Fourier transform infra-red (FTIR) spectroscopic analysis}

The FT-IR spectrometer (model IR-Prestige-21 Shimadzu Corporation, Kyoto, Japan) was used to record the spectra of the oil samples. A drop of each sample was poured between two disks of $\mathrm{KBr}$ to prepare a thin film. The spectra were scanned throughout the region from $4000 \mathrm{~cm}^{-1}$ to $400 \mathrm{~cm}^{-1}$ and recorded as percent transmittance values (Zahir et al., 2017).

\subsection{Statistical analysis}

The statistical analysis of the experimental data was done by taking a triplicate measurement of the sets for all parameters and the values were reported in terms of mean \pm standard deviation (SD) in the 
respective tables. Comparison of the mean values was made using analysis of variance (ANOVA) followed by Tukey's test on SPSS 15.0 at $\alpha=0.05$ significant level.

\section{RESULTS AND DISCUSSION}

The physicochemical properties of oils after treatments of conventional heating and microwave heating are reported in Tables 1 and 2. The results showed changes in physicochemical properties of edible oils brought on by the application of conventional and microwave heating.

\subsection{Effect of heating on the density and viscosity of edible oil}

The density and viscosity data explain the physical nature of oils. The presence of double bonds and length of the hydrocarbon chain in the triglyceride molecule affect the density and viscosity of oils. The values for densities and viscosities of the oil samples were decreased after conventional heating (Adejumo et al., 2015), while they were increased after microwave heating ( $\mathrm{Li}$ et al., 2018). It was observed that corn oil had lower values of density and viscosity as compared to soybean oil. The higher amount of polyunsaturated fatty acids (linoleic acid) of corn oil is responsible for lower density and viscosity values (Abbas et al., 2016). Soybean oil had higher viscosity values due to the presence of less unsaturated fatty acids as compared to corn oil. After conventional heating, the kinetic energy of the oils was increased and the degradation of oil molecules takes place, which lowers the oil viscosity (Diamante and Lan, 2014). After the application of microwave heating, these unsaturated fatty acids were converted into high-molecular-weight polymeric compounds, cyclic compounds, dimers, trimers, and epoxides, resulting in the formation of a large molecule and led to an increase in viscosity values (Srivastava and Semwal, 2015; Li et al., 2018).

\subsection{Effect of heating treatment on acid value}

The acid content of the oil is the measurement of fatty acids in oils. It was observed that with conventional heating, acid values were increased by $21.20 \%$ and $32.29 \%$ for corn oil and soybean oil, respectively. The same results were observed for the microwave heating of the oils for up to $1 \mathrm{~min}$. Microwave heating at a higher temperature, with an increased exposure time of up to $10 \mathrm{~min}$ caused a $10.61 \%$ and a $44.56 \%$ decrease in the acid values of corn and soybean oil, respectively. Corn oil had a high amount of free fatty acids, as observed by the high

TABLE 1. Impact of conventional heating on the oxidative properties of corn oil and soybean oil

\begin{tabular}{|c|c|c|c|c|c|c|c|c|c|}
\hline $\begin{array}{l}\text { Temperature } \\
\left({ }^{\circ} \mathbf{K}\right)\end{array}$ & $\begin{array}{c}\mathrm{d} \\
\times 10^{3}\left(\mathrm{~kg} / \mathrm{m}^{3}\right)\end{array}$ & $\begin{array}{c}\eta \\
\mathbf{x 1 0}^{4} \\
\text { (Pa.s) }\end{array}$ & $\begin{array}{c}\mathrm{AV} \\
(\mathrm{mg} / \mathrm{g})\end{array}$ & $\begin{array}{c}\mathrm{PV} \\
\left(\mathrm{meq} \mathrm{O}_{2} / \mathrm{kg}\right)\end{array}$ & $\begin{array}{c}\mathrm{SV} \\
(\mathrm{mg} / \mathrm{g})\end{array}$ & p-AV & CDs & CTs & TOTOX \\
\hline \multicolumn{10}{|l|}{ Corn Oil } \\
\hline 303 & $\begin{array}{r}0.9080^{\mathrm{e}} \\
\pm 0.0001\end{array}$ & $\begin{array}{l}35.741^{\mathrm{e}} \\
\pm 0.203\end{array}$ & $\begin{array}{r}0.316^{\mathrm{a}} \\
\pm 0.011\end{array}$ & $\begin{array}{l}34.610^{\mathrm{a}} \\
\pm 0.300\end{array}$ & $\begin{array}{l}159.67^{\mathrm{e}} \\
\pm 2.517\end{array}$ & $\begin{array}{r}6.271^{\mathrm{a}} \\
\pm 0.204\end{array}$ & $\begin{array}{r}1.605^{\mathrm{a}} \\
\pm 0.100\end{array}$ & $\begin{array}{r}1.493^{\mathrm{a}} \\
\pm 0.127\end{array}$ & $\begin{array}{l}75.491^{\mathrm{a}} \\
\pm 0.802\end{array}$ \\
\hline 313 & $\begin{array}{c}0.9041^{\mathrm{d}} \\
\pm 0.0002\end{array}$ & $\begin{array}{c}33.985^{\mathrm{d}} \\
\pm 0.418\end{array}$ & $\begin{array}{r}0.325^{\mathrm{b}} \\
\pm 0.011\end{array}$ & $\begin{array}{l}41.090^{\mathrm{b}} \\
\pm 0.207\end{array}$ & $\begin{array}{l}145.00^{\mathrm{d}} \\
\pm 1.000\end{array}$ & $\begin{array}{r}6.815^{\mathrm{b}} \\
\pm 0.400\end{array}$ & $\begin{array}{c}1.643^{\mathrm{b}} \\
\pm 0.100\end{array}$ & $\begin{array}{r}1.573^{\mathrm{b}} \\
\pm 0.112\end{array}$ & $\begin{array}{c}88.995^{\mathrm{b}} \\
\pm 0.113\end{array}$ \\
\hline 323 & $\begin{array}{r}0.9004^{c} \\
\pm 0.0004\end{array}$ & $\begin{array}{l}33.557^{c} \\
\pm 0.177\end{array}$ & $\begin{array}{r}0.348^{\mathrm{c}} \\
\pm 0.011\end{array}$ & $\begin{array}{l}44.580^{c} \\
\pm 0.205\end{array}$ & $\begin{array}{l}130.42^{\mathrm{c}} \\
\pm 1.506\end{array}$ & $\begin{array}{r}7.474^{\mathrm{c}} \\
\pm 0.402\end{array}$ & $\begin{array}{r}1.674^{\mathrm{c}} \\
\pm 0.100\end{array}$ & $\begin{array}{r}1.623^{\mathrm{c}} \\
\pm 0.129\end{array}$ & $\begin{array}{l}96.634^{\circ} \\
\pm 0.811\end{array}$ \\
\hline 333 & $\begin{array}{r}0.8963^{\mathrm{b}} \\
\pm 0.0001\end{array}$ & $\begin{array}{l}32.090^{\mathrm{b}} \\
\pm 0.204\end{array}$ & $\begin{array}{r}0.369^{\mathrm{d}} \\
\pm 0.010\end{array}$ & $\begin{array}{l}46.390^{\mathrm{d}} \\
\pm 0.300\end{array}$ & $\begin{array}{l}122.00^{\mathrm{b}} \\
\pm 1.000\end{array}$ & $\begin{array}{r}7.918^{\mathrm{d}} \\
\pm 0.200\end{array}$ & $\begin{array}{r}1.693^{\mathrm{d}} \\
\pm 0.225\end{array}$ & $\begin{array}{r}1.709^{\mathrm{d}} \\
\pm 0.051\end{array}$ & $\begin{array}{l}100.70^{\mathrm{d}} \\
\pm 0.800\end{array}$ \\
\hline 343 & $\begin{array}{r}0.8908^{\mathrm{a}} \\
\pm 0.0002\end{array}$ & $\begin{array}{l}31.655^{\mathrm{a}} \\
\pm 0.413\end{array}$ & $\begin{array}{r}0.383^{\mathrm{e}} \\
\pm 0.010\end{array}$ & $\begin{array}{l}50.670^{\mathrm{e}} \\
\pm 0.350\end{array}$ & $\begin{array}{l}117.49^{\mathrm{a}} \\
\pm 0.500\end{array}$ & $\begin{array}{r}8.248^{\mathrm{e}} \\
\pm 0.104\end{array}$ & $\begin{array}{r}1.822^{\mathrm{e}} \\
\pm 0.101\end{array}$ & $\begin{array}{r}1.759^{\mathrm{e}} \\
\pm 0.060\end{array}$ & $\begin{array}{l}109.59^{\mathrm{e}} \\
\pm 0.802\end{array}$ \\
\hline \multicolumn{10}{|l|}{ Soybean Oil } \\
\hline 303 & $\begin{array}{r}0.9093^{\mathrm{a}} \\
\pm 0.0004\end{array}$ & $\begin{array}{l}37.690^{\mathrm{a}} \\
\pm 0.107\end{array}$ & $\begin{array}{r}0.213^{\mathrm{a}} \\
\pm 0.010\end{array}$ & $\begin{array}{c}27.047^{\mathrm{d}} \\
\pm 0.450\end{array}$ & $\begin{array}{l}183.75^{\mathrm{a}} \\
\pm 1.010\end{array}$ & $\begin{array}{r}6.061^{\mathrm{b}} \\
\pm 0.402\end{array}$ & $\begin{array}{r}1.342^{\mathrm{a}} \\
\pm 0.179\end{array}$ & $\begin{array}{r}1.586^{\mathrm{c}} \\
\pm 0.112\end{array}$ & $\begin{array}{l}60.141^{\mathrm{a}} \\
\pm 1.300\end{array}$ \\
\hline 313 & $\begin{array}{r}0.9049^{\mathrm{a}} \\
\pm 0.0001\end{array}$ & $\begin{array}{c}35.890^{\mathrm{b}} \\
\pm 0.351\end{array}$ & $\begin{array}{r}0.249^{\mathrm{b}} \\
\pm 0.011\end{array}$ & $\begin{array}{l}37.959^{\mathrm{c}} \\
\pm 0.401\end{array}$ & $\begin{array}{l}181.00^{\mathrm{a}} \\
\pm 2.082\end{array}$ & $\begin{array}{r}6.163^{\mathrm{b}} \\
\pm 0.402\end{array}$ & $\begin{array}{r}1.399^{\mathrm{a}} \\
\pm 0.057\end{array}$ & $\begin{array}{r}1.825^{\mathrm{b}} \\
\pm 0.101\end{array}$ & $\begin{array}{c}82.081^{\mathrm{b}} \\
\pm 1.205\end{array}$ \\
\hline 323 & $\begin{array}{r}0.9007^{\mathrm{b}} \\
\pm 0.0003\end{array}$ & $\begin{array}{l}34.573^{\mathrm{c}} \\
\pm 0.404\end{array}$ & $\begin{array}{r}0.250^{\mathrm{b}} \\
\pm 0.010\end{array}$ & $\begin{array}{l}40.860^{\mathrm{b}} \\
\pm 0.203\end{array}$ & $\begin{array}{r}179.00^{\mathrm{a}, \mathrm{b}} \\
\pm 1.523\end{array}$ & $\begin{array}{l}6.796^{\mathrm{a}, \mathrm{b}} \\
\pm 0.016\end{array}$ & $\begin{array}{l}1.454^{\mathrm{a}, \mathrm{b}} \\
\pm 0.100\end{array}$ & $\begin{array}{r}1.834^{\mathrm{b}} \\
\pm 0.045\end{array}$ & $\begin{array}{l}88.516^{\mathrm{c}} \\
\pm 0.422\end{array}$ \\
\hline 333 & $\begin{array}{l}0.8977^{\mathrm{b}, \mathrm{c}} \\
\pm 0.0011\end{array}$ & $\begin{array}{c}33.196^{\mathrm{d}} \\
\pm 0.251\end{array}$ & $\begin{array}{l}0.261^{\mathrm{b}, \mathrm{c}} \\
\pm 0.010\end{array}$ & $\begin{array}{l}39.391^{\mathrm{a}} \\
\pm 0.400\end{array}$ & $\begin{array}{r}175.00^{\mathrm{b} . c} \\
\pm 2.523\end{array}$ & $\begin{array}{r}7.232^{\mathrm{a}} \\
\pm 0.300\end{array}$ & $\begin{array}{r}1.539^{\mathrm{b}} \\
\pm 0.053\end{array}$ & $\begin{array}{r}1.863^{\mathrm{b}} \\
\pm 0.019\end{array}$ & $\begin{array}{c}86.014^{\mathrm{d}} \\
\pm 1.100\end{array}$ \\
\hline 343 & $\begin{array}{r}0.8949^{c} \\
\pm 0.0002\end{array}$ & $\begin{array}{l}32.075^{\mathrm{e}} \\
\pm 0.356\end{array}$ & $\begin{array}{r}0.282^{\mathrm{c}} \\
\pm 0.011\end{array}$ & $\begin{array}{l}41.694^{\mathrm{a}} \\
\pm 0.472\end{array}$ & $\begin{array}{l}173.00^{\mathrm{c}} \\
\pm 2.001\end{array}$ & $\begin{array}{r}7.566^{\mathrm{a}} \\
\pm 0.200\end{array}$ & $\begin{array}{r}1.632^{\mathrm{b}} \\
\pm 0.050\end{array}$ & $\begin{array}{r}2.079^{\mathrm{a}} \\
\pm 0.053\end{array}$ & $\begin{array}{l}90.954^{\mathrm{e}} \\
\pm 0.758\end{array}$ \\
\hline
\end{tabular}

The data are reported as the average of triplicates $(\mathrm{N}=3), \pm$ standard deviation. The significant mean difference is indicated by different letters $(\mathrm{a}, \mathrm{b}, \mathrm{c}, \mathrm{d}, \mathrm{e})(\mathrm{P} \leq 0.05)$ according to Tukey test. d density, $\eta$ viscosity, AV acid value, $\mathrm{PV}$ peroxide value, SV saponification value, $\rho-\mathrm{AV}$ para-anisidine value, CDs conjugated dienes, CTs conjugated trienes, TOTOX total oxidation value. 
TABLE 2. Impact of microwave heating on the oxidative properties of corn oil and soybean oil

\begin{tabular}{|c|c|c|c|c|c|c|c|c|c|}
\hline $\begin{array}{l}\text { Heating time } \\
\text { (min) }\end{array}$ & $x 10^{3}\left(\mathrm{~kg} / \mathrm{m}^{3}\right)$ & $\begin{array}{c}\eta \\
\mathbf{x 1 0 ^ { 4 }} \\
\text { (Pa. s) }\end{array}$ & $\underset{(\mathrm{mg} / \mathrm{g})}{\mathrm{AV}}$ & $\begin{array}{c}\mathrm{PV} \\
\left(\mathrm{meq} \mathrm{O}_{2} / \mathrm{kg}\right)\end{array}$ & $\underset{(\mathrm{mg} / \mathrm{g})}{\mathrm{SV}}$ & $\mathbf{p}-\mathbf{A V}$ & CDs & CTs & TOTOX \\
\hline \multicolumn{10}{|l|}{ Corn Oil } \\
\hline 0.5 & $\begin{array}{r}0.9046^{\mathrm{c}} \\
\pm 0.0003\end{array}$ & $\begin{array}{l}27.866^{\mathrm{d}} \\
\pm 0.105\end{array}$ & $\begin{array}{r}1.007^{\mathrm{a}} \\
\pm 0.006\end{array}$ & $\begin{array}{l}70.470^{\mathrm{a}} \\
\pm 0.104\end{array}$ & $\begin{array}{l}146.11^{\mathrm{c}} \\
\pm 3.772\end{array}$ & $\begin{array}{l}13.377^{\mathrm{f}} \\
\pm 0.089\end{array}$ & $\begin{array}{l}13.536^{\mathrm{c}} \\
\pm 0.002\end{array}$ & $\begin{array}{r}1.412^{\mathrm{f}} \\
\pm 0.001\end{array}$ & $\begin{array}{l}154.32^{\mathrm{a}} \\
\pm 0.085\end{array}$ \\
\hline 1 & $\begin{array}{r}0.9055^{\mathrm{c}} \\
\pm 0.0002\end{array}$ & $\begin{array}{l}31.453^{\mathrm{c}} \\
\pm 1.209\end{array}$ & $\begin{array}{r}1.140^{\mathrm{a}} \\
\pm 0.501\end{array}$ & $\begin{array}{r}50.00^{\mathrm{b}} \\
\pm 1.001\end{array}$ & $\begin{array}{r}181.00^{\mathrm{b}, \mathrm{c}} \\
\pm 1.326\end{array}$ & $\begin{array}{l}47.510^{\mathrm{e}} \\
\pm 0.100\end{array}$ & $\begin{array}{r}12.56^{\mathrm{d}} \\
\pm 0.003\end{array}$ & $\begin{array}{r}2.205^{\mathrm{e}} \\
\pm 0.002\end{array}$ & $\begin{array}{l}147.51^{\mathrm{b}} \\
\pm 2.025\end{array}$ \\
\hline 2 & $\begin{array}{r}0.9071^{\mathrm{c}} \\
\pm 0.0003\end{array}$ & $\begin{array}{l}35.075^{\mathrm{b}} \\
\pm 0.111\end{array}$ & $\begin{array}{r}1.111^{\mathrm{a}} \\
\pm 0.050\end{array}$ & $\begin{array}{r}6.167^{\mathrm{c}} \\
\pm 0.253\end{array}$ & $\begin{array}{r}213.08^{\mathrm{a}, \mathrm{b}} \\
\pm 1.781\end{array}$ & $\begin{array}{l}90.973^{\mathrm{d}} \\
\pm 0.042\end{array}$ & $\begin{array}{r}10.78^{\mathrm{e}} \\
\pm 0.001\end{array}$ & $\begin{array}{r}4.173^{\mathrm{b}} \\
\pm 0.003\end{array}$ & $\begin{array}{l}103.31^{\mathrm{c}} \\
\pm 0.231\end{array}$ \\
\hline 4 & $\begin{array}{r}0.9100^{\mathrm{b}} \\
\pm 0.0006\end{array}$ & $\begin{array}{l}36.355^{\mathrm{b}} \\
\pm 0.150\end{array}$ & $\begin{array}{r}1.087^{\mathrm{a}} \\
\pm 0.054\end{array}$ & $\begin{array}{r}5.803^{\mathrm{c}} \\
\pm 0.614\end{array}$ & $\begin{array}{r}242.08^{\mathrm{a}, \mathrm{b}} \\
\pm 1.211\end{array}$ & $\begin{array}{l}77.127^{\mathrm{c}} \\
\pm 0.196\end{array}$ & $\begin{array}{r}13.67^{\mathrm{c}} \\
\pm 0.004\end{array}$ & $\begin{array}{r}4.442^{\mathrm{a}} \\
\pm 0.005\end{array}$ & $\begin{array}{c}88.733^{\mathrm{d}} \\
\pm 0.738\end{array}$ \\
\hline 6 & $\begin{array}{l}0.9132^{\mathrm{a}, \mathrm{b}} \\
\pm 0.0004\end{array}$ & $\begin{array}{l}37.983^{\mathrm{a}} \\
\pm 0.500\end{array}$ & $\begin{array}{r}1.027^{\mathrm{a}} \\
\pm 0.650\end{array}$ & $\begin{array}{r}5.293^{\mathrm{c}} \\
\pm 0.507\end{array}$ & $\begin{array}{l}272.76^{\mathrm{a}} \\
\pm 2.661\end{array}$ & $\begin{array}{l}71.200^{\mathrm{b}} \\
\pm 0.087\end{array}$ & $\begin{array}{r}17.83^{\mathrm{b}} \\
\pm 0.005\end{array}$ & $\begin{array}{r}3.802^{\mathrm{c}} \\
\pm 0.002\end{array}$ & $\begin{array}{l}81.786^{\mathrm{e}} \\
\pm 0.178\end{array}$ \\
\hline 10 & $\begin{array}{r}0.9167^{\mathrm{a}} \\
\pm 0.0002\end{array}$ & $\begin{array}{l}39.058^{\mathrm{a}} \\
\pm 0.350\end{array}$ & $\begin{array}{r}1.019^{\mathrm{a}} \\
\pm 0.011\end{array}$ & $\begin{array}{r}5.074^{\mathrm{c}} \\
\pm 0.161\end{array}$ & $\begin{array}{l}293.76^{\mathrm{a}} \\
\pm 1.361\end{array}$ & $\begin{array}{l}65.360^{\mathrm{a}} \\
\pm 0.197\end{array}$ & $\begin{array}{r}20.00^{\mathrm{a}} \\
\pm 0.082\end{array}$ & $\begin{array}{r}3.544^{\mathrm{d}} \\
\pm 0.004\end{array}$ & $\begin{array}{l}75.508^{\mathrm{f}} \\
\pm 0.034\end{array}$ \\
\hline \multicolumn{10}{|l|}{ Soybean Oil } \\
\hline 0.5 & $\begin{array}{r}0.9059^{\mathrm{d}} \\
\pm 0.0001\end{array}$ & $\begin{array}{l}27.470^{\mathrm{f}} \\
\pm 1.001\end{array}$ & $\begin{array}{r}1.305^{\mathrm{c}} \\
\pm 0.400\end{array}$ & $\begin{array}{l}47.193^{\mathrm{a}} \\
\pm 0.622\end{array}$ & $\begin{array}{l}72.059^{\mathrm{f}} \\
\pm 2.462\end{array}$ & $\begin{array}{l}12.032^{\mathrm{f}} \\
\pm 0.117\end{array}$ & $\begin{array}{r}7.089^{\mathrm{a}} \\
\pm 0.000\end{array}$ & $\begin{array}{r}1.059^{\mathrm{e}} \\
\pm 0.001\end{array}$ & $\begin{array}{l}106.42^{\mathrm{b}} \\
\pm 0.285\end{array}$ \\
\hline 1 & $\begin{array}{l}0.9060^{\mathrm{c}, \mathrm{d}} \\
\pm 0.0003\end{array}$ & $\begin{array}{l}29.219^{\mathrm{e}} \\
\pm 1.105\end{array}$ & $\begin{array}{r}2.078^{\mathrm{a}} \\
\pm 0.371\end{array}$ & $\begin{array}{l}45.455^{\mathrm{b}} \\
\pm 0.478\end{array}$ & $\begin{array}{l}100.20^{\mathrm{e}} \\
\pm 3.356\end{array}$ & $\begin{array}{l}25.047^{\mathrm{e}} \\
\pm 0.230\end{array}$ & $\begin{array}{l}6.4301^{\mathrm{d}} \\
\pm 0.032\end{array}$ & $\begin{array}{r}1.436^{\mathrm{d}} \\
\pm 0.000\end{array}$ & $\begin{array}{l}115.91^{\mathrm{a}} \\
\pm 0.818\end{array}$ \\
\hline 2 & $\begin{array}{r}0.9069^{c} \\
\pm 0.0002\end{array}$ & $\begin{array}{l}31.793^{\mathrm{d}} \\
\pm 0.114\end{array}$ & $\begin{array}{r}1.846^{\mathrm{b}} \\
\pm 0.103\end{array}$ & $\begin{array}{l}7.4725^{\mathrm{c}} \\
\pm 0.155\end{array}$ & $\begin{array}{l}113.52^{\mathrm{d}} \\
\pm 2.756\end{array}$ & $\begin{array}{c}63.386^{\mathrm{d}} \\
\pm 0.589\end{array}$ & $\begin{array}{r}6.623^{\mathrm{c}} \\
\pm 0.005\end{array}$ & $\begin{array}{r}3.518^{\mathrm{a}} \\
\pm 0.003\end{array}$ & $\begin{array}{l}78.377^{\mathrm{f}} \\
\pm 0.201\end{array}$ \\
\hline 4 & $\begin{array}{r}0.9088^{\mathrm{b}} \\
\pm 0.0004\end{array}$ & $\begin{array}{l}33.609^{c} \\
\pm 0.357\end{array}$ & $\begin{array}{r}1.279^{\mathrm{c}} \\
\pm 0.052\end{array}$ & $\begin{array}{l}6.9444^{\mathrm{d}} \\
\pm 0.692\end{array}$ & $\begin{array}{l}124.93^{\mathrm{b}} \\
\pm 2.200\end{array}$ & $\begin{array}{l}63.835^{\mathrm{c}} \\
\pm 0.196\end{array}$ & $\begin{array}{r}6.613^{\mathrm{c}} \\
\pm 0.003\end{array}$ & $\begin{array}{l}3.470^{\mathrm{a}, \mathrm{b}} \\
\pm 0.002\end{array}$ & $\begin{array}{l}77.715^{\mathrm{e}} \\
\pm 0.742\end{array}$ \\
\hline 6 & $\begin{array}{l}0.9100^{\mathrm{a}} \\
\pm 0.005\end{array}$ & $\begin{array}{c}35.552^{\mathrm{b}} \\
\pm 0.450\end{array}$ & $\begin{array}{c}1.184^{\mathrm{d}} \\
\pm 0.400\end{array}$ & $\begin{array}{r}6.6556^{\mathrm{d}, \mathrm{e}} \\
\pm 0.001\end{array}$ & $\begin{array}{l}135.49^{\mathrm{a}} \\
\pm 2.224\end{array}$ & $\begin{array}{c}70.828^{\mathrm{b}} \\
\pm 0.299\end{array}$ & $\begin{array}{c}6.421^{\mathrm{d}} \\
\pm 0.001\end{array}$ & $\begin{array}{r}3.279^{\mathrm{c}} \\
\pm 0.001\end{array}$ & $\begin{array}{c}84.273^{\mathrm{d}} \\
\pm 0.203\end{array}$ \\
\hline 10 & $\begin{array}{r}0.9112^{\mathrm{a}} \\
\pm 0.0007\end{array}$ & $\begin{array}{l}37.222^{\mathrm{a}} \\
\pm 0.202\end{array}$ & $\begin{array}{r}1.152^{\mathrm{d}} \\
\pm 0.450\end{array}$ & $\begin{array}{l}6.5445^{\mathrm{e}} \\
\pm 0.216\end{array}$ & $\begin{array}{l}121.18^{\mathrm{c}} \\
\pm 0.011\end{array}$ & $\begin{array}{c}76.539^{\mathrm{a}} \\
\pm 0.151\end{array}$ & $\begin{array}{r}6.707^{\mathrm{b}} \\
\pm 0.002\end{array}$ & $\begin{array}{c}3.4339^{\mathrm{b}} \\
\pm 0.001\end{array}$ & $\begin{array}{l}89.771^{c} \\
\pm 0.504\end{array}$ \\
\hline
\end{tabular}

The data are reported as the average of triplicates $(\mathrm{N}=3), \pm$ standard deviation. The significant mean difference is indicated by different letters ( $\mathrm{a}, \mathrm{b}, \mathrm{c}, \mathrm{d}, \mathrm{e}, \mathrm{f})(\mathrm{P} \leq 0.05)$ according to Tukey test. $\mathrm{d}$ density, $\eta$ viscosity, AV acid value, $\mathrm{PV}$ peroxide value, $\mathrm{SV}$ saponification value, p-AV para-anisidine value, CDs conjugated dienes, CTs conjugated trienes, TOTOX total oxidation value.

acid values. During repeated conventional heating, the degradation of triglycerides and the formation of fatty acids were observed by increased acid values (Halim et al., 2016; Adejumo et al., 2015). With microwave heating, the amount of fatty acids increased (Taghvaei et al., 2014) and then these fatty acids degraded to hydro peroxides which were further converted to secondary oxidation products and lower acid values (Leong et al., 2015). The acid values for corn oil showed greater degradation with microwave radiation exposure compared to soybean oil, which confirmed the oxidative stability of soybean oil (Adejumo et al., 2015).

\subsection{Saponification value}

Oils with high saponification values are considered to be better edible sources which contain high proportions of low-molecular-weight and shortchain fatty acids (Adejumo et al., 2015; Hussain et al., 2015). The saponification value of soybean oil was higher compared to corn oil with conventional heating from $303{ }^{\circ} \mathrm{K}$ to $323^{\circ} \mathrm{K}$. Microwave heating $(0.5 \mathrm{~min}$ to $10 \mathrm{~min})$ increased the temperature from
$343{ }^{\circ} \mathrm{K}$ to $543{ }^{\circ} \mathrm{K}$ compared to conventional heating. The lower saponification values for soybean oil with microwave heating compared to corn oil indicated less formation of short- chain fatty acids. The higher degradation rate of long-chain fatty acids to short-chain fatty acids is responsible for higher saponification values in corn oil (Rutckeviski et al., 2016). It was concluded that conventional heating of oil samples converts the triglyceride molecules to glycerol and fatty acids which showed a $35.90 \%$ and $5.46 \%$ decrease in saponification values for corn oil and soybean oil, respectively. However, significant increases in saponification values for corn oil $(101.05 \%)$ and soybean oil $(68.17 \%)$ were observed with microwave heating due to an increase in the amount of short-chain fatty acids (Rutckeviski et al., 2016).

\subsection{Peroxide value}

The peroxide value is the chemical property used to measure the extent of rancidity in oils. Fresh oil has lower peroxide values compared to oxidized oil. The results tabulated in Tables 1 and 2 reveal 
that the peroxide values for corn oil are high compared to soybean oil. The conventional heating of oil showed a $46.40 \%$ and $54.14 \%$ increase in the amount of hydro peroxides due to the process of oxidation of fatty acids. The same observation was made for canola seed oil (Jalili et al., 2017). The peroxide values of microwave heated oil were initially increased upon irradiation but the continuous exposure of microwave irradiation showed $92.79 \%$ and $86.13 \%$ decreases in peroxide values with increased heating time, which resulted in the formation of secondary oxidation products by the breaking of hydro peroxides (Abbas et al., 2016; Ghosh et al., 2014).

\section{5. $P$-anisidine value}

The peroxide value cannot be used to determine the rancidity of oil at higher heating temperatures due to the instability of hydro peroxides. Various volatile and non-volatile compounds, including dimers, trimers, alcohols, polymers, and other secondary oxidation products are produced by the degradation of hydro peroxides, which strongly affects the stability of the oil and makes it more rancid. This fact led to the representation of rancidity of oil in terms of the $p$-anisidine value ( $p-\mathrm{AV})$ and total oxidation value (TOTOX value). The $p$-anisidine value of edible oil samples determines the amount of hydro peroxides as well as the aldehydic and ketonic forms of products (Leong et al., 2015). During conventional heating, $p$-anisidine values were increased by $31.53 \%$ and $24.83 \%$ for corn oil and soybean oil, respectively. Microwave heating showed a $536.13 \%$ increase in the $p$-anisidine values in soybean oil; whereas the $p$-anisidine values in the corn oil samples were initially increased by $580.0 \%$ (Ghosh et al., 2014) and then further heating caused a $256.82 \%$ decrease. $P$-anisidine values were high for microwave irradiated oil samples compared to conventional heated oil samples, which showed more chemical deterioration in oils due to exposure to microwave irradiation (Abbas et al., 2016).

\subsection{Total oxidation value}

The total oxidation value (TOTOX) expresses the oxidation state of the oil. The lower value of total oxidation signified better nutritional value of the oil. TOTOX values were calculated by the equation (Halim et al., 2016);

TOTOX value $=p-\mathrm{AV}+(2 * \mathrm{PV})$

The results reported in Tables 1 and 2 reveal that the soybean oil samples had low TOTOX value compared to the corn oil. The lower TOTOX values for soybean oil indicated higher nutritional value than corn oil. It was also observed that corn oil showed $45.17 \%$ and $51.07 \%$ increases in TOTOX values, whereas soybean oil showed increases of $51.23 \%$ and $15.64 \%$ with conventional and microwave heating, respectively. It was also noticed that oil samples exposed to microwave irradiation had higher TOTOX values compared to the conventionally heated oil samples. (Abbas et. al., 2016). The effect of conventional heating $\left(343^{\circ} \mathrm{K}\right)$ and microwave heating $(0.5 \mathrm{~min})$ on the oxidative properties of oils are represented in Figure 2.

\subsection{UV-spectroscopic indices}

UV-spectroscopic indices in terms of conjugated dienes (CDs) and conjugated trienes (CTs) were monitored at $232 \mathrm{~nm}$ and $268 \mathrm{~nm}$, respectively. The conjugated dienes (CDs) and conjugated trienes (CTs)

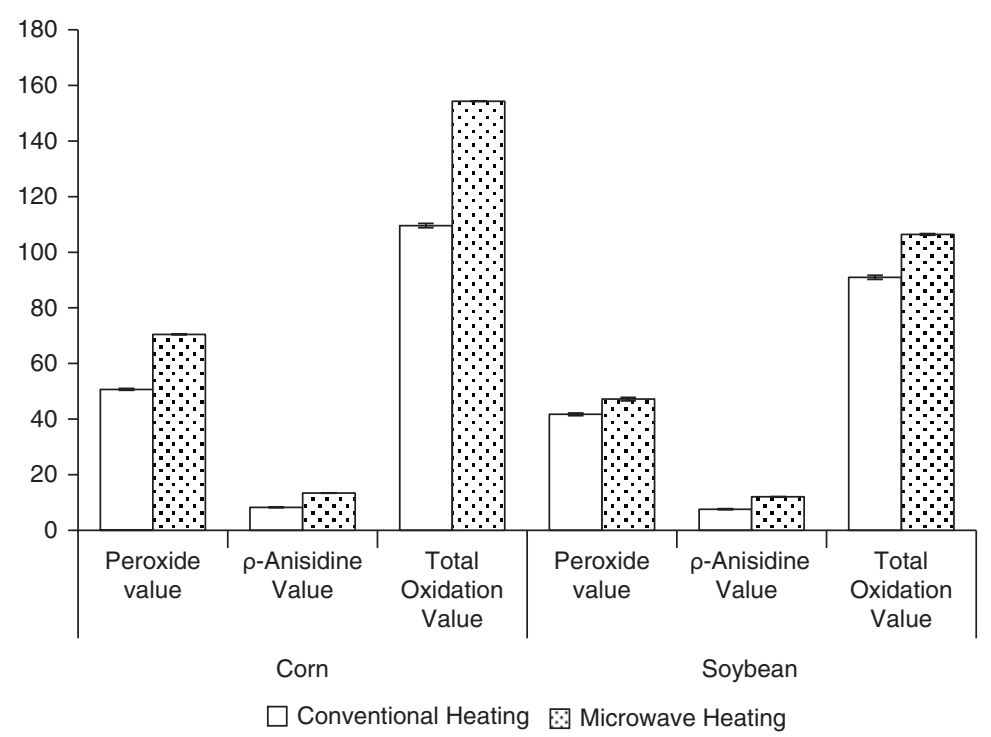

FIGURE 2. Effect of conventional heating $\left(343^{\circ} \mathrm{K}\right)$ and microwave heating time $(0.5 \mathrm{~min})$ on oxidative properties of corn and soybean oil. Data are presented as the mean of triplicates with error bars denoting standard deviation. 
in the oil were generated by the rearrangement of double bond positions in the monounsaturated fatty acids and polyunsaturated fatty acids, respectively $(\mathrm{Li}$ et al., 2018). Oils that have low mono and polyunsaturated fatty acids exhibit low formation of conjugated dienes and conjugated trienes, respectively. Increased values of CDs and CTs upon conventional and microwave heating, indicated the degradation of hydro peroxides and formation of conjugated dienes and conjugated trienes for both oils. Corn oil showed greater values of CDs and low values of CTs compared to the soybean oil. Corn oil contains a high amount of monounsaturated fatty acids, so, the formation of dienes was higher, which was confirmed by greater values of CDs (Ghosh et al., 2014). The conventional heating of corn and soybean oil showed $13.52 \%$ and $21.61 \%$ increases; whereas microwave heating showed $47.75 \%$ and $5.38 \%$ increases in $C D$ values, respectively. On the other hand, the conventional heating of corn and soybean oil showed $17.82 \%$ and $31.08 \%$ increases in CT values; whereas microwave heating showed $150.99 \%$ and $224.26 \%$ increases in CT values, respectively. A previous report on Perah seed oil (Li et al., 2018) and pumpkin seed oil (Abbas et al., 2017) also confirmed the significant increase in CDs and CTs with increasing heating periods. The oxidative stability of oil decreased with the increase in UV-spectroscopic indices of oils. The low CD and CT values for soybean oil mean better oxidative stability upon conventional and microwave heating.

\subsection{Fourier transform infra-red spectroscopy}

The Fourier Transform Infra-Red Spectroscopy analysis was used to study the oxidative rancidity of oils. Figures 3 and 4 show the FT-IR spectra of corn oil and soybean oil, respectively, with different heating

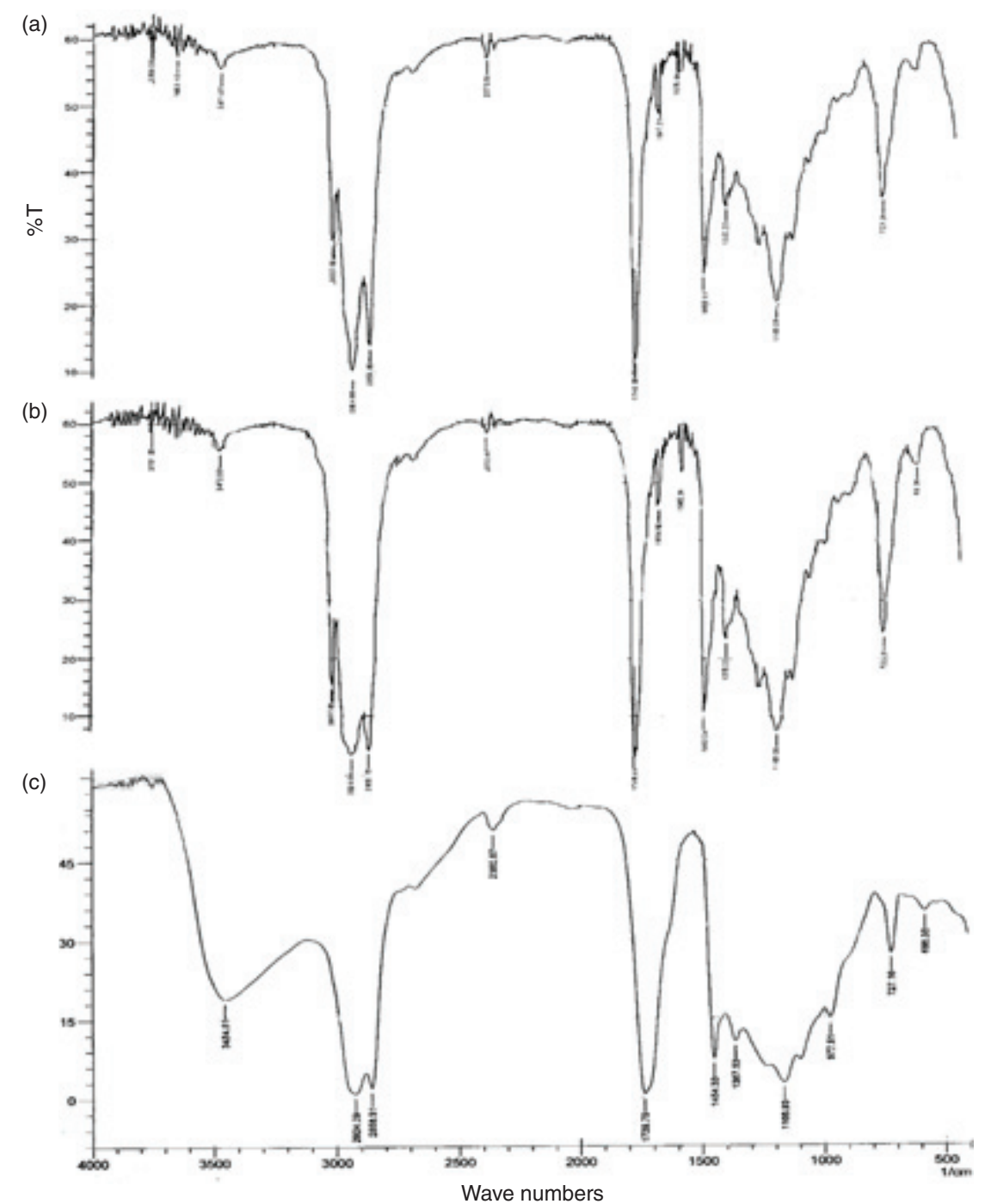

FIGURE 3. FTIR spectra of corn oil (a) fresh oil, (b) conventional heating at $343^{\circ} \mathrm{K}$, (c) microwave heating for $10 \mathrm{~min}$. 


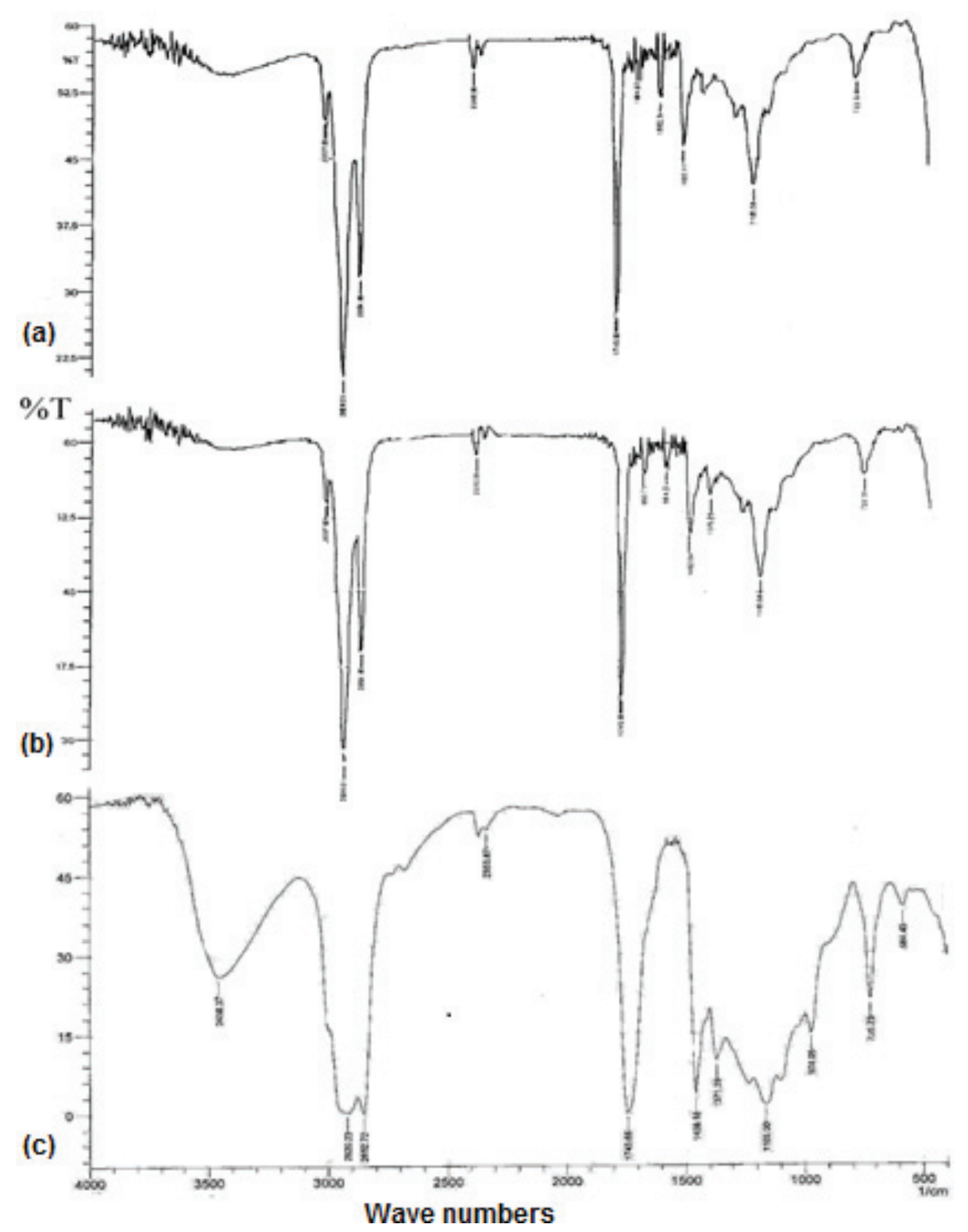

FIGURE 4. FTIR spectra of soybean oil, (a) fresh oil, (b) conventional heating at $343^{\circ} \mathrm{K}$, (c) microwave heating for $10 \mathrm{~min}$.

treatments. During the continuous heating process, the hydrolysis of oil takes place, which resulted in an increase in the amount of hydro peroxides, free fatty acids, mono glycerides, and diglycerides. The formation of secondary oxidation products and characterization of the oils were made through the intensities and frequencies of the peaks and bands in the FT-IR spectra (Zahir et al., 2017; Sadoudi et al., 2014).

The assignment of the vibrations of functional groups in the infrared spectra of the oils are as follows: $3471.87-3441.01 \mathrm{~cm}^{-1}(\mathrm{OH}$ stretching vibration of hydro peroxides and FFAs of $\equiv \mathrm{C}-\mathrm{H}), 3100-3000$ $\mathrm{cm}^{-1}$ (strong peak of $-\mathrm{CH}\left(\mathrm{sp}^{2}\right.$ and $\mathrm{sp}$ ) stretching vibration of cis-double bonds in unsaturated fatty acids) (linoleic and linoleic acyl group), 3000-2850 $\mathrm{cm}^{-1}$ (Two peaks of $\mathrm{CH}_{3}$ symmetric stretching and $\mathrm{sp}^{2} \mathrm{CH}_{2}$ asymmetric stretching of aldehyde, which confirms the presence of aldehydes), $2679.13 \mathrm{~cm}^{-1}$ (-C=O fermi resonance) (Wu et al., 2015), $1745 \mathrm{~cm}^{-1}$ (aliphatic ester $-\mathrm{C}=\mathrm{O}$ stretching in the carbonyl group of triglycerides, which means the oil has high nutritional value) (Srivastava et al., 2015).

The FT-IR spectra of heated oil samples show a peak at around $3300 \mathrm{~cm}^{-1}$, which indicates the formation of hydro peroxides, as observed by Srivastava et al., 2015. The intensity of the cis-double bond near $3008 \mathrm{~cm}^{-1}$ remained almost unaltered or suffered a very slow shifting towards smaller values during oxidative stress. The bands at $2927 \mathrm{~cm}^{-1}$ and 2854 $\mathrm{cm}^{-1}$ increased their absorbance due to surrounding chemical changes as a consequence of the oxidation process (Abbas et al., 2017). Intensity at $1745 \mathrm{~cm}^{-1}$ 
was increased on heating as hydro peroxides decomposed to carbonyl compounds which are secondary oxidation products (carbonyl compounds i.e. aldehydes, ketones, dimers, trimers etc.) and shifted the peak towards a lower frequency $\sim 1728 \mathrm{~cm}^{-1}$ (Srivastava et al., 2015). The smallest variations in the intensities at $2854 \mathrm{~cm}^{-1}$ and $1745 \mathrm{~cm}^{-1}$ were observed for conventional heating, which was justified by the small variations in $\mathrm{CD}$ and $\mathrm{CT}$ values. Similar observations were reported by Srivastava et al., 2015 in heated virgin coconut oil. The intensity of the band near $1465 \mathrm{~cm}^{-1}$ tended to increase with the oxidative treatment. These increments were found to be higher in the microwave heated oil, indicating that oxidation proceeded more slowly in the conventional heated oil samples compared to the microwave heated one (Abbas et al., 2017).

\subsection{The degree of chain length and degree of branching}

The peak heights of IR bands were used to calculate some ratios for the comparison of spectra. A symmetric deformation of $\mathrm{CH}_{3}$ and $\mathrm{CH}_{2}+\mathrm{CH}_{3}$ appeared at $1375 \mathrm{~cm}^{-1}$ and at $1460 \mathrm{~cm}^{-1}$, respectively. The measurement of the degree of branching was made by the ratios of $A_{1375} / A_{1460}$. The degree of

TABLE 3. Impact of conventional heating and microwave heating on the degree of chain length and degree of branching of corn oil and soybean oil

\begin{tabular}{|c|c|c|c|}
\hline \multirow{2}{*}{$\begin{array}{l}\text { Temperature } \\
\left({ }^{\circ} \mathbf{K}\right)\end{array}$} & \multicolumn{2}{|c|}{ Degree of Chain Length } & \multirow{2}{*}{$\begin{array}{c}\text { Degree of } \\
\text { Branching } \\
\mathrm{A}_{1375} / \mathrm{A}_{1460} \\
\end{array}$} \\
\hline & $\mathbf{A}_{723} / \mathbf{A}_{1375}$ & $\mathbf{A}_{723} / \mathbf{A}_{1460}$ & \\
\hline & \multicolumn{3}{|c|}{ Corn Oil (Conventional heating) } \\
\hline 303 & 0.9714 & 0.6410 & 0.6599 \\
\hline 323 & 0.9659 & 0.6320 & 0.6446 \\
\hline \multirow[t]{2}{*}{343} & 0.9591 & 0.6304 & 0.6239 \\
\hline & \multicolumn{3}{|c|}{ Soybean Oil (Conventional heating) } \\
\hline 303 & 0.9742 & 0.7091 & 0.6692 \\
\hline 323 & 0.9682 & 0.694 & 0.6535 \\
\hline 343 & 0.9607 & 0.6754 & 0.6381 \\
\hline $\begin{array}{l}\text { Heating time } \\
(\mathrm{min})\end{array}$ & \multicolumn{3}{|c|}{ Corn Oil (Microwave heating) } \\
\hline 0.5 & 0.6037 & 0.4559 & 0.7208 \\
\hline 1 & 0.6057 & 0.4735 & 0.7318 \\
\hline 4 & 0.6591 & 0.4923 & 0.7469 \\
\hline \multirow[t]{2}{*}{10} & 0.6802 & 0.4965 & 0.7558 \\
\hline & \multicolumn{3}{|c|}{ Soybean Oil (Microwave heating) } \\
\hline 0.5 & 0.5843 & 0.4417 & 0.6363 \\
\hline 1 & 0.5899 & 0.4529 & 0.6502 \\
\hline 4 & 0.6420 & 0.4677 & 0.6787 \\
\hline 10 & 0.6656 & 0.4699 & 0.6986 \\
\hline
\end{tabular}

chain length was measured by the ratio of absorbance band of $\mathrm{CH}_{2}$ rocking vibration at $720 \mathrm{~cm}^{-1}$ and $\mathrm{CH}_{2}+\mathrm{CH}_{3}$ or $\mathrm{CH}_{3}$ symmetric deformation at $1375 \mathrm{~cm}^{-1}$ and $1460 \mathrm{~cm}^{-1}$, respectively, or $\mathrm{A}_{720} / \mathrm{A}_{1460}$ or $\mathrm{A}_{720} / \mathrm{A}_{1375}$ (El-Bassoussi et al., 2010).

The degree of branching and chain length was measured for all treated oil samples and reported in Table 3 for conventional and microwave heating. The results showed that the degree of branching and the degree of chain length of the fatty acids of the oils decreased as the oil was conventionally heated. Microwave irradiation increased the branching pattern and chain length of fatty acids. The results also revealed that the ratios of $\mathrm{A}_{1375} / \mathrm{A}_{1460}$ had higher values for soybean oil compared to corn oil samples.

\section{CONCLUSIONS}

This study concerned the comparison of two heating techniques (conventional and microwave) on the basis of oxidative properties. Microwave heating may be easier and time-saving but it destroys the nutritional values of food, confirmed by observing the physiochemical properties of oils. The oxidative stability of corn oil and soybean oil was investigated using both techniques. Conventional heating was found to be a safer method for the preparation of food as less degradation of primary oxidation products was observed compared to microwave heating. It was concluded that microwave heating increased the degradation of hydro peroxides and the formation of secondary oxidation products which decreased the nutritional values of oils. The physicochemical parameters of the heated oil samples showed that the oxidation rate of the fatty acids in corn oil was higher compared to the soybean oil, which was also proven through FTIR spectra. On the basis of the results, it was concluded that the use of microwave heating depreciates the characteristics of oils and should be minimized for cooking food. Soybean oil exhibits more oxidative stability compared to corn oil and is better to be used as an edible source. The present study can be extended to evaluate the oxidative stability of oils upon frying with different food items.

\section{REFERENCES}

Abbas AM, Nargis A, Othman NH, Noor AF, Sadik G, Hossen J. 2017. Oxidation stability and compositional characteristics of oils from microwave roasted pumpkin seeds during thermal oxidation. Inter. J. Food Prop. 20, 2569-2580. https://doi.org/10.1080/10942912.2016.1244544

Abbas AM, Hadi Bin Mesran M, Latip RA, Hidayu ON, Mahmood NAN. 2016. Effect of microwave heating with different exposure times on the degradation of corn oil. Int. Food Res. J. 23, 842-848.

Adejumo BA, Olorunsogo ST, Omosaiye SI. 2015. Qualities of tiger nut oil as influenced by heating temperature. Int. J. Emer. Technol. Eng. 2, 2348-8050

Aluyor EO, Ori-Jesu. 2008. The use of antioxidants in vegetable oils - A review. Afr. J. Biotechnol. 7, 4836-4842. 
AOCS. 1997. Official methods and recommended practices of the American Oil Chemists' Society. 5th edition, Champaign, USA: AOCS Press.

AOCS. 1993. Official methods and recommended practices of the American Oil Chemists' Society. Champaign, USA: AOCS Press; 762.

Aydınkaptan E, Mazı IB. 2017. Monitoring the physicochemical features of sunflower oil and French fries during repeated microwave frying and deep-fatfrying. Grasas Aceites $\mathbf{6 8}$, 1-12. https://doi.org/10.3989/gya.1162162

Bakhshabadi H, Mirzaei H, Ghodsvali A, Jafari SM, Ziaiifar AM, Farzaneh V. 2017. The effect of microwave pretreatment on some physico-chemical properties and bioactivity of Black cumin seeds' oil. Ind. Crop. Prod. 97, 1-9. https:// doi.org/10.1016/j.indcrop.2016.12.005

Bakhshabadi H, Mirzaei H, Ghodsvali A, Jafari SM, Ziaiifar AM. 2018. The influence of pulsed electric fields and microwave pretreatments on some selected physicochemical properties of oil extracted from black cumin seed. Food Sci. Nutr. 6, 111-118. https://doi.org/10.1002/fsn3.535

Caponio F, Pasqualone A, Gomes T. 2003. Changes in the fatty acid composition of vegetable oils in model doughs submitted to conventional or microwave heating. Int. J. Food Sci. Tech. 38, 481-486. https://doi. org/10.1046/j.1365-2621.2003.00703.x

Diamante LM, Lan T. 2014. Absolute viscosities of vegetable oils at different temperatures and shear rate range of 64.5 to $4835 \mathrm{~s}^{-1}$. J. Food Process. 2014, 1-6. https://doi. org/10.1155/2014/234583

El-Bassoussi AA, Ahmed MHM, Sayed SE, Basta JS, Attia SE. 2010. Characterization of some local petroleum residues by spectroscopic techniques. Petroleum Sci. Tech. 28, 430-444. https://doi.org/10.1080/10916460902744554

El-Hadad N, Abou-Gharbia HA, Ei-Aal MH, Youssef MM. 2010. Red Palm Olein: Characterization and Utilization in Formulating Novel Functional Biscuits. J. Am. Oil Chem. Soc. 87, 295-304. https://doi.org/10.1007/s11746-009-1497-x

Fasina OO, Hallman CHM, Clementsa C. 2006. Predicting Temperature-Dependence Viscosity of Vegetable Oils from Fatty Acid Composition. J. Am. Oil Chem. Soc. 83, 899-903. https://doi.org/10.1007/s11746-006-5044-8

Ghosh J, Banerjee A, Gupta SS, Sengupta A, Ghosh M. 2014. Comparative degradation effects of sesame and soybean oil during heating using microwave irradiation. J. Sci. Ind. Res. 73, 547-552.

Halim Y, Natania, Halim MJ, Soedirga CL, Yakhin AL. 2016. Physical and chemical characteristics of frying oil in Indonesia in a repeated frying model. J. Chem. Pharm. Res. 8, 583-589.

Hussain R, Hussain A, Asadullah, Sattar S, Zeb M, Hussain A, Nafees M. 2015. Physico-chemical properties and assessment of edible oil potential of peanuts grown in Kurram Agency, Parachinar. Pak. J. Anal. Environ. Chem. 16, 45-51.

Jalili F, Jafari SM, Emam-Djomeh Z, Malekjani N, Farzaneh V. 2017. Optimization of ultrasound-assisted extraction of oil from canola seeds with the use of response surface methodology. Food Anal. Methods 11, 598-612. http://doi. org/10.1007/s12161-017-1030-z

Leong XF, Ng CY, Jaarin K, Mustafa MR. 2015. Effects of repeated heating of cooking oils on antioxidant content and endothelial function. Austin J. Pharmacol. Ther. 3, $1-7$.

Li SK, Ali AM, Muhammad II, Othman HN, Noor MA. 2018. The effect of microwave roasting over the thermooxidative degradation of Perah seed oil during heating. J. Oleo Sci. 67, 497-505. https://doi.org/10.5650/jos. ess 17203

Lukešová D, Dostálová J, Mahmoud EAE, Svárovská M. 2009. Oxidation changes of vegetable oils during microwave heating. Czech J. Food Sci. 27, S178-S181. https://doi. org/10.17221/929-CJFS

Rafiee Z, Jafari SM, Alami M, Khomeiri M. 2012. Antioxidant effect of microwave-assisted extracts of olive leaves on sunflower oil. J. Agric. Sci. Technol. 14, 1497-1509.

Rutckeviski R, Xavier-Júnior FH, Morais ARV, Alencar EN, Amaral-Machado L, Genre J, Gondim AD, Egito EST. 2017. Thermo-Oxidative Stability Evaluation of Bullfrog (Rana catesbeiana Shaw) Oil. Molecules 22, 606-621. https://doi.org/10.3390/molecules22040606

Sadoudi R, Ammouche A, Ahmed DA. 2014. Thermal oxidative alteration of sunflower oil. Afr. J. Food Sci. 8, 116-121. https://doi.org/10.5897/AJFS12.112

Saeed GMS, Sayeed AS, Ashraf S, Saify SZ, Ali R, Zareen T, Haider S. 2014. Effect of FDA approved edible dyes on physicochemical stability of microwave treated olive oil. J. Adv. Chem. 10, 3274-3283.

Srivastava Y, Semwal AD. 2015. A study on monitoring of frying performance and oxidative stability of virgin coconut oil (VCO) during continuous/prolong deep fat frying process using chemical and FTIR spectroscopy. J. Food Sci. Technol. 52, 984-991. https://doi.org/10.1007/ s13197-013-1078-8

Taghvaei M, Jafari SM, Assadpoor E, Nowrouzieh S, Alishah O. 2014. Optimization of microwave-assisted extraction of cottonseed oil and evaluation of its oxidative stability and physicochemical properties. Food Chem. 160, 90-97. https://doi.org/10.1016/j.foodchem.2014.03.064

Taghvaei M, Jafari SM, Nowrouzieh S, Alishah O. 2015. The influence of cooking process on the microwave-assisted extraction of cottonseed oil. J. Food Sci. Technol. 52, 1138-1144. https://doi.org/10.1007/s13197-013-1125-5

Wu Z, Li H, Tu D. 2015. Application of Fourier Transform Infrared (FT-IR) Spectroscopy combined with chemometrics for analysis of rapeseed oil adulterated with refining and purificating waste cooking oil. Food Anal. Methods 8, 2581-2587. https://doi.org/10.1007/ s12161-015-0149-z

Zahir E, Saeed R, Hameed MA, Yousuf A. 2017. Study of physicochemical properties of edible oil and evaluation of frying oil quality by Fourier transform-infrared (FT-IR) spectroscopy. Arab. J. Chem. 10, S3870-S3876. https://doi. org/10.1016/j.arabjc.2014.05.025 